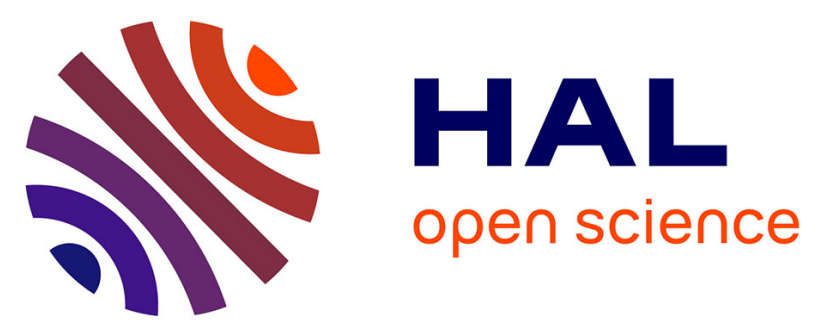

\title{
Demonstration of optically controlled data routing with the use of multiple-quantum-well bistable and electro-optical devices
}

Pàl Koppa, Pierre Chavel, Jean-Louis Oudar, Robert Kuszelewicz, Jean-Philippe Schnell, Jean-Paul Pocholle

\section{To cite this version:}

Pàl Koppa, Pierre Chavel, Jean-Louis Oudar, Robert Kuszelewicz, Jean-Philippe Schnell, et al.. Demonstration of optically controlled data routing with the use of multiple-quantum-well bistable and electro-optical devices. Applied optics, 1997, 36 (23), pp.5706-5716. 10.1364/AO.36.005706 . hal-00879012

\section{HAL Id: hal-00879012 \\ https://hal-iogs.archives-ouvertes.fr/hal-00879012}

Submitted on 31 Oct 2013

HAL is a multi-disciplinary open access archive for the deposit and dissemination of scientific research documents, whether they are published or not. The documents may come from teaching and research institutions in France or abroad, or from public or private research centers.
L'archive ouverte pluridisciplinaire HAL, est destinée au dépôt et à la diffusion de documents scientifiques de niveau recherche, publiés ou non, émanant des établissements d'enseignement et de recherche français ou étrangers, des laboratoires publics ou privés. 


\title{
Demonstration of optically controlled data routing with the use of multiple-quantum-well bistable and electro-optical devices
}

\author{
P. Koppa, P. Chavel, J. L. Oudar, R. Kuszelewicz, J. P. Schnell, and J. P. Pocholle
}

\begin{abstract}
We present experimental results on a 1-to-64-channel free-space photonic switching demonstration system based on GaAs/GaAlAs multiple-quantum-well active device arrays. Two control schemes are demonstrated: data transparent optical self-routing usable in a packet-switching environment and direct optical control with potential signal amplification for circuit switching. The self-routing operation relies on the optical recognition of the binary destination address coded in each packet header. Address decoding is implemented with elementary optical bistable devices and modulator pixels as all-optical latches and electro-optical AND gates, respectively. All 60 defect-free channels of the system could be operated one by one, but the simultaneous operation of only three channels could be achieved mainly because of the spatial nonhomogeneities of the devices. Direct-control operation is based on directly setting the bistable device reflectivity with a variable-control beam power. This working mode turned out to be much more tolerant of spatial noises: 37 channels of the system could be operated simultaneously. Further development of the system to a crossbar of $N$ inputs and $M$ outputs and system miniaturization are also considered. (C) 1997 Optical Society of America

Key words: Free-space photonic switching, self-routing, address recognition, optical logic, spatial light modulators, optical bistable devices.
\end{abstract}

\section{Introduction}

The needs of telecommunication and computing systems for reconfigurable high-bandwidth parallel interconnects have triggered intense research in freespace photonic switching. Significant progress has been made in the investigation of network topologies and routing methods that are suitable for optical implementation. In parallel, spectacular results have been achieved in device development, especially in the fields of semiconductor and liquid-crystal devices.

Two basic approaches are present in the system conception: One approach considers optics a means

When this research was performed, P. Koppa and P. Chavel were with the Institut d'Optique, Centre National de la Recherche Scientifique (CNRS), BP 147, 91403 Orsay Cedex, France. J. L. Oudar and R. Kuszelewicz were with France Telecom CNET Paris-B, BP 107, 92225 Bagneux Cedex, France. J. P. Schnell and J. P. Pocholle were with Thomson CSF, LCR, Domaine de Corbeville, 91404 Orsay Cedex, France. P. Koppa is now with the Department of Atomic Physics, Technical University of Budapest, Budafoki út 8, H-1111 Budapest, Hungary.

Received 17 September 1996; revised manuscript received 12 March 1997.

0003-6935/97/235706-11 $\$ 10.00 / 0$

(C) 1997 Optical Society of America for interconnecting more or less complex electronic islands, while the other approach aims at maintaining the optical continuity of the signals, avoiding the optic-to-electronic and electronic-to-optic conversions. A few successful demonstrator systems have been described recently for the first approach with arrays of smart pixels. ${ }^{1}$ Demonstrations of the second concept often involve guided-wave devices, ${ }^{2}$ but there exist a few experimental results in free-space optics as well. 3,4

We attempt to prove the validity of a novel freespace switching concept with optical continuity of the signals and optical control of the network. Two control schemes are proposed and demonstrated: optical self-routing for packet switching and simple direct optical addressing with potential signal reshaping and amplification. The self-routing operation requires a complex node functionality, the decoding of a binary header address that is commonly implemented by electronic switches and smart pixels but is unusual with all-optical devices. This operation is accomplished here by means of optical bistable devices that perform the logical operations of address identification, switching, and memorization of the switch state. Either a spatial light modulator or a 
microlaser array can be used to introduce the reference addresses of each output channel.

To demonstrate this concept, we built a 1-input to 64-output switch with an $8 \times 8$ array of electroabsorption modulators and a planar optical bistable cavity. Both devices are based on GaAs/GaAlAs quantum-well structures. A brief description of the active device properties is given in Section 2, with an emphasis on the most critical parameters such as spatial uniformities, contrast ratios, hysteresis width, and wavelength compatibility. In Section 3 the operating principle of the control schemes implemented in packet-switching and circuit-switching environments are presented in detail. In Section 4 experimental work on the setup is outlined and the main experimental results obtained during the test of the system are presented. In Section 5 the system we generalize to $N \times M$ crossbar switches that can be used individually or as building blocks for multistage interconnection networks. A possible miniaturized version of the system is also proposed in that section.

\section{Description of Active Devices}

\section{A. Optical Bistable Device}

A reflection-type nonlinear Fabry-Perot etalon is considered as a four-port device with two inputs and two outputs (Fig. 1). One of the inputs, called the holding or control beam, provides a constant bias power to maintain the device at a suitable working point. The other input port is used to introduce data in the form of one or more superimposed signal beams. The two possible outputs are the reflected control beam and the reflected signal beam $(\mathrm{s})$. We can change the optical response (reflected power versus input power) of a nonlinear Fabry-Perot cavity from a continuous function through a steplike function to a hysteresis curve by increasing the initial detuning from the Fabry-Perot resonance [Fig. $1(b)] .5$

The bistable device used in this experiment is a planar (nonpixellated) Fabry-Perot cavity with a multiple-quantum-well (MQW) active medium stacked between two Bragg reflectors. The front and back mirrors consist of, respectively, 23.5 and 17 periods of alternating $\mathrm{AlAs}$ and $\mathrm{Ga}_{0.9} \mathrm{Al}_{0.1}$ As quarterwave layers, while the active region includes 19 pairs of $10-\mathrm{nm}$ thin $\mathrm{Ga}_{0.5} \mathrm{Al}_{0.5} \mathrm{As}$ barriers and $10-\mathrm{nm}$ thin GaAs quantum wells. The measured finesse of the cavity is 380 . By using an incident light spot of 26 $\mu \mathrm{m}$ and a $1 / e^{2}$ diameter, we measured a bistability threshold of $\sim 3 \mathrm{~mW}$, which corresponded to a threshold illumination of $6 \mu \mathrm{W} / \mu \mathrm{m}^{2}$.

One of the most critical parameters of the entire experiment is the spatial uniformity of the switching thresholds of the bistable device. In a Fabry-Perot bistable device, the switching thresholds depend strongly on wavelength detuning from the resonance, as is depicted in Fig. 1(b). The higher the finesse, the greater the dependence. ${ }^{6}$ Hence, when one works at constant wavelength, any variation of the etalon thickness will be turned into switching thresh-

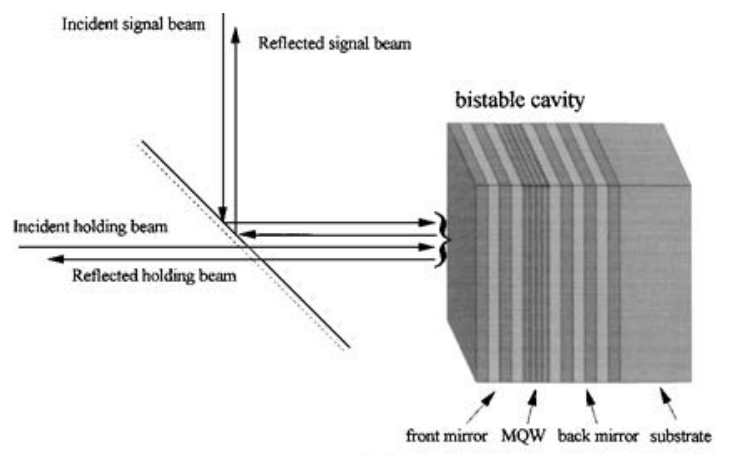

(a)
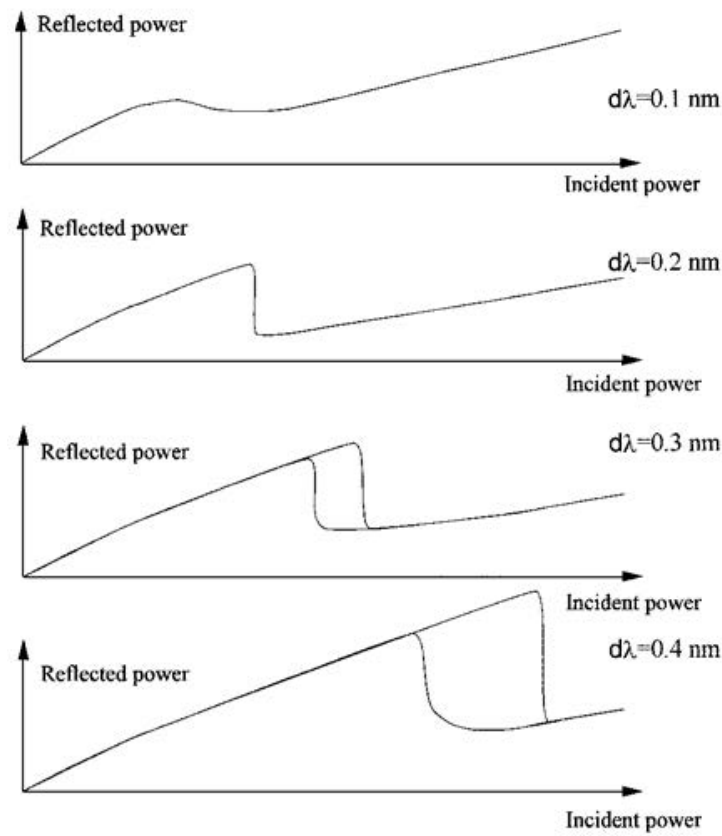

(b)

Fig. 1. Reflection-type nonlinear Fabry-Perot resonator with (a) input and output signals and (b) response curves at four different values of cavity detuning from the Fabry-Perot resonance.

old fluctuation. One can distinguish between long(millimeter-) and short- (micrometer-) scale fluctuations.

The long-scale threshold variation is influenced mainly by a slight decrease in the layer thicknesses going from the center of the wafer to the border. As a consequence the resonance wavelength of the cavity varies from $864 \mathrm{~nm}$ at the sample center to $850 \mathrm{~nm}$ at the border. This long-scale variation does not constitute a fundamental limitation to the device: with appropriate molecular organic vacuum phase epitaxy reactors some groups have obtained a growth uniformity better than $0.1 \%$ over an entire wafer. ${ }^{7}$ To some extent, the consequences of the long-scale thickness variations can also be reduced with a smaller wafer area, i.e., smaller and more closely packed pixels. Etched microcavities ${ }^{8}$ specifically present efficient carrier and light confinement within a pixel, so the separation distance between two adjacent pixels can be as low as a few micrometers, and light can be 


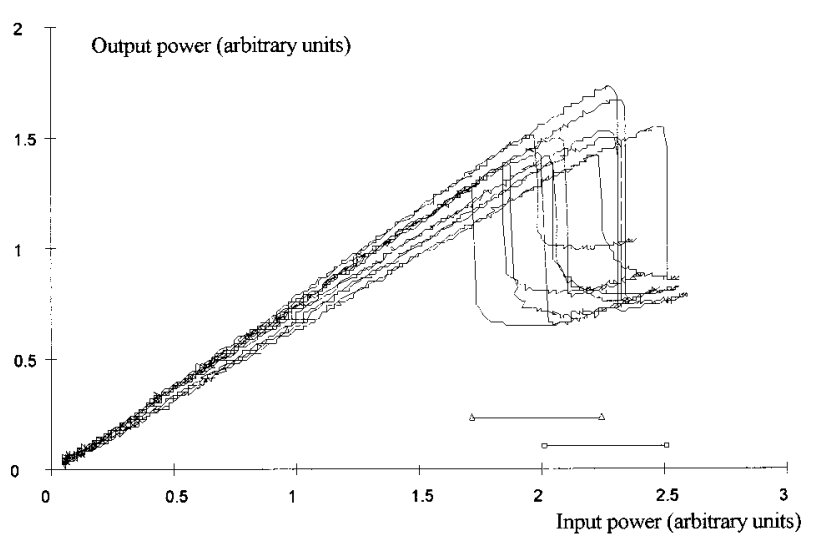

Fig. 2. Eight superimposed response curves of the bistable cavity used. The two markers under the curves illustrate the spread of the switch ON and the switch OFF thresholds for this set of bistables.

coupled into the microresonators by use of largenumerical-aperture optics. The threshold dispersion introduced by the pixellation process is under investigation. Note that the lateral confinement is a very efficient method of threshold reduction as well. Threshold powers below $100 \mu \mathrm{W}$ have been achieved with the samples presented in Ref. 9.

In addition to the systemic threshold variation, short-scale random threshold fluctuations have to be considered. ${ }^{10}$ These fluctuations are due to local defects and interface roughness determined by the growth process at a microscopic scale. If the typical size of these defects exceeds a few micrometers, the threshold uniformity may be affected dramatically by an interface roughness of one or two atomic monolayers. If the correlation length of these defects stays significantly below the spot size they will not degrade the threshold uniformity.

As an example, Fig. 2 shows eight superimposed response curves that correspond to some of the elementary bistable spots of the $8 \times 8$ array with a spacing of $60 \mu \mathrm{m}$, as is used in the switching experiments. It is unfortunate that on our present sample there is a surface defect just in the wafer center, so the array has to be placed $\sim 1 \mathrm{~mm}$ away from this point. The threshold variations shown are greater than the mean hysteresis width, so the bistable regions do not overlap. The typical threshold nonuniformity of the whole $8 \times 8$ array is in excess of $\pm 20 \%$, while the hysteresis width is approximately $15 \%$. The uniformity of the device reflectivities is estimated to be $\pm 10 \%$. A more recent device based on a bulk AlGaAs active layer rather than MQW's presented somewhat lower threshold fluctuations $( \pm 15 \%)$ and a much larger hysteresis width $(50 \%)$. For this device the superposed hysteresis curves do overlap.

The switching contrast ratio of the device used in the experiment is approximately 2. Significantly higher contrast ratios (approximately 30) have been achieved with other samples, ${ }^{11}$ but their use with our present modulator arrays is not possible because of wavelength incompatibility.
Temporal changes of the bistable device properties can arise mainly from temperature drifts. The temperature variation of the threshold powers is approximately $25 \% /{ }^{\circ} \mathrm{C}$ in the bistable range that is used for the latching operation. Consequently, a temperature stability of better than $\pm 0.04{ }^{\circ} \mathrm{C}$ is required to maintain the threshold values constant within $\pm 1 \%$. This can be achieved by an electronically controlled Peltier cooler, but local heating effects can still occur in the bistable device if the total input power is too large.

\section{B. Electroabsorption Modulator Array}

An array of $8 \times 8$ individually addressable electroabsorption modulators is used to introduce the 64 reference addresses in the system. The modulator element is a GaAs/GaAlAs p-i-n mesa structure with 130 quantum wells in the intrinsic region. ${ }^{12}$ The transmission of the device is modulated by the absorption changes that occur owing to the quantumconfined Stark effect. ${ }^{13}$ At room temperature and a wavelength of $860 \mathrm{~nm}$, the transmission of the device varies typically from $25 \%$ to $5 \%$ when the voltage over the quantum-well structure is slowly increased from 0 to $19 \mathrm{~V}$. This means a dc modulation contrast of $\sim 5$. A contrast ratio of 3.5-4 is obtained by use of a more convenient voltage swing of $6 \mathrm{~V}$, between 6 and $12 \mathrm{~V}$. The circular pixels have a diameter of $100 \mu \mathrm{m}$ and a pitch of $300 \mu \mathrm{m}$. The rise and fall times are $\mathrm{RC}$ limited to $\sim 1 \mathrm{~ns}$. In the matrix used in the experiment, 60 of the 64 pixels were operating correctly; one did not present any modulation, and the remaining three could not be controlled individually because their driving electrodes were accidentally linked together.

A considerably degraded contrast ratio was obtained for high-speed modulation: at $20 \mathrm{MHz}$ the contrast was less than 2 . In the same time, the optimal operation wavelength was shifted to $866 \mathrm{~nm}$. We can explain this degradation by driving the device at intermediate frequencies: On a $1-\mathrm{KHz}$ square wave two distinct transition times can be observed clearly. For example, when the driving voltage falls from 12 to $6 \mathrm{~V}$, there is a fast rise in the transmission in $\sim 6 \mathrm{~ns}$, corresponding to the electrical time constant of the driving circuits; then the transmission continues to increase very slowly, with a 5-orders-ofmagnitude longer rise time of $\sim 500 \mu \mathrm{s}$. The contrast ratio of the fast modulation is between 1.5 and 2 ; but with the addition of the slower one, a dc contrast of 4 is obtained. We suspect the origin of the slow transition to be thermal for two reasons: first, the effect does not appear at very low optical powers, and, second, its time constant is of the same order as the characteristic time of the heat exchanges of a pixel with its environment.

The displacement of the operating wavelength at higher bit rates can also be explained by the local elevation of the temperature. This effect can be very annoying if the device is to be used at variable power, frequency, or duty cycle. (In particular, the duty cycle of the signals can fluctuate in asynchronous 


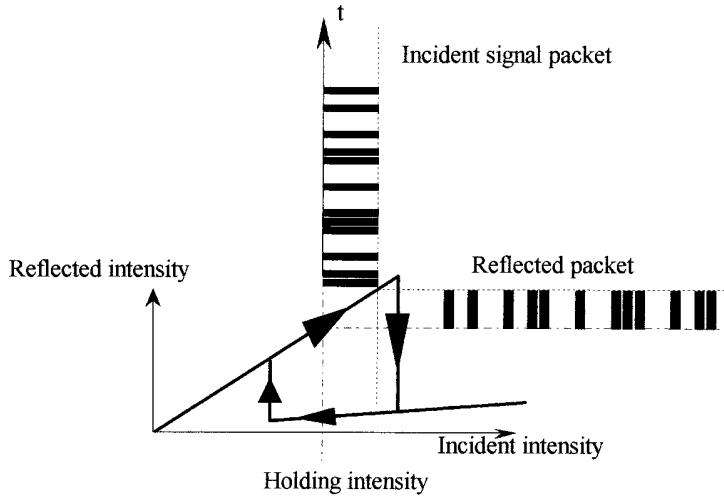

(a)

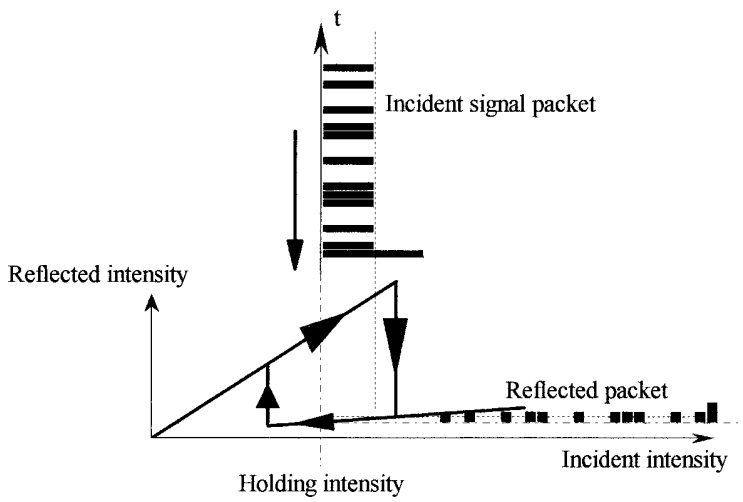

(b)

Fig. 3. Latching operation of a bistable device. The device is initially set in the high-reflectivity state within the hysteresis region: (a) The signal beam is transmitted when no switching pulse arrives. (b) The signal beam is absorbed by the substrate when there is a header pulse that exceeds the switch-OFF threshold.

packet-switching networks, in which the arrival of the packets is random.) The remedy to this problem can be the use of reflection-type modulators to improve heat sinking through the substrate.

In our present devices the substrate was removed to realize optical access from both sides; therefore heat conduction could take place only within the thin layer of the devices. On the other hand, the contrast ratio can also be increased, largely by use of reflection-type modulators with an asymmetric Fabry-Perot structure. ${ }^{14}$ Wafer homogeneity is of the same order of the bistable cavities: Our best modulator array presented $\pm 17 \%$ transmission fluctuations at a wavelength of $860 \mathrm{~nm}$ when it was set in the high transmission state with a dc voltage of $6 \mathrm{~V}$.

\section{Data Switching with Bistable Devices}

\section{A. Packet Switching}

In the packet-switching mode, self-routing is implemented on the basis of the latching operation of bistable devices and optical decoding of the header address that precedes each data packet. The latching of a bistable device is shown in Fig. 3: We initially set the device in the high-reflectivity state within the

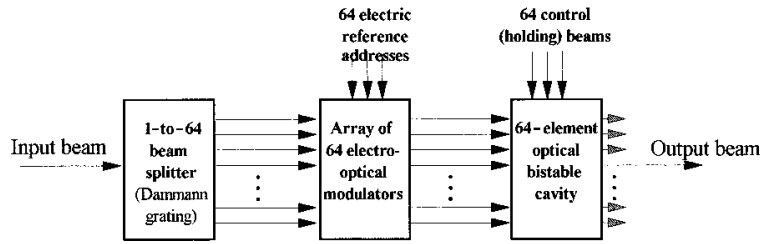

(a)
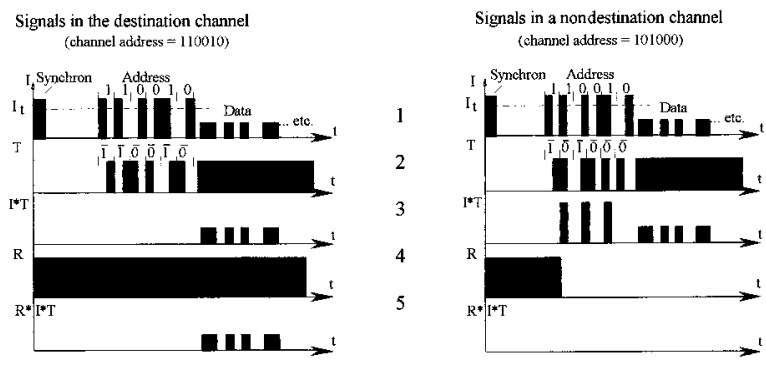

(b)

Fig. 4. Block diagram of the experimental setup: (a) Signal diagram of the address-decoding operation. (b) 1, input signal; 2 , transmission of the modulator element; 3 , signal transmitted by the modulator; 4 , reflectivity of the corresponding bistable element; 5 , signal reflected by the bistable element (equals the output).

hysteresis region of the bistable response curve by use of a holding beam. The device can be switched down by a switching pulse, provided that the sum of the switching and the holding powers exceeds the higher threshold. Signal pulses emitted at a power level lower than the threshold will not switch the latch, so the blocking or passing state of the switch will be determined by the presence or absence of the high-power switching pulse in the packet header. To reset the latch, one must decrease the control beam below the hysteresis region. This kind of latching can be used directly for self-routing switching, as was shown by Buller et al. ${ }^{15}$

In our approach the packet header contains a binary header address; therefore an additional operation, decoding of the destination address, is needed in each of the 64 channels of the system. This addressdecoding operation has to produce a suitable switching pulse on each bistable device, i.e., no high-level pulse for the destination channel and one or more high-level switching pulses for the other channels. To implement this operation by simple optical means, we used the so-called Manchester coding technique for the binary addresses: Each bit is followed by its complementary value, so bit 1 is encoded by a highlow sequence and bit 0 by a low-high one. By use of this code, address comparison becomes a simple bitwise multiplication of the packet address by the inversely coded channel address.

The operation is presented in Fig. 4 in the example of two channels: One is the destination channel and the other is an arbitrary nondestination channel. First, the input signal beam is divided into 64 subbeams, so that each subbeam illuminates one elementary modulator of the $8 \times 8$ array. By this fan-out operation, all modulator elements receive the same signal 
at the same instant: first a synchronisation bit, then the 6-bit destination address, and finally the data payload at lower amplitude [see the first row of Fig. 4(b)]. In the example of the figure, the destination address of 50 (binary 110010) is emitted with the above-mentioned code. The synchronizing pulse of the arriving packet triggers each modulator pixel to deliver its own address in inverse coding (high-low sequence is for bit 0 ; low-high is for bit 1 ).

This modulation must be in perfect synchronizm with the arrival of the destination address on the modulator array [see row 2 of Fig. 4(b)]. In this way the emerging power from each modulator element is the bitwise product of two sequences, the Manchester-coded destination address and the inversely coded address of the channel in question [see row 3 of Fig. 4(b)]. If and only if the two sequences are complementary, which is equivalent to the identity of the two addresses, there is no high-level light pulse passing through the modulator pixel at any time during address emission. However, any mismatch between the two addresses is turned into the transmission of a high-level switching pulse by the modulator element. (Note that simple nonreturnto-zero encoding the address comparision could not be done because only the faulty 1's, not the 0's, of the destination address would give a switching pulse. For the modulator array that is imaged upon the bistable array, each bistable element receives light from the corresponding modulator pixel. Consequently, the only bistable element that stays in the reflective state will be that of the destination channel, where address matching is perfect [row 4 of Fig. 4(b)]. The data packet passes through this channel without triggering the switching of the bistable device, given that the data amplitude is lower than the switching threshold. After the packet has passed, all bistable devices are reset to the initial high-reflection state to receive the next packet. This reset operation generates dead time of the order of 1 address bit period.

\section{B. Circuit Switching}

The simplest operating mode possible is one that controls the reflectivity of the cavity directly by the control-beam power, as shown in Fig. 4. In this scheme the steplike response curve of the nonlinear Fabry-Perot cavity is used [Fig. 1(b), curve with 0.2 $\mathrm{nm}$ detuning) and the modulator array is placed on the holding-beam branch in order to obtain individual control of holding beams. When the control beam is off [Fig. 5(a)], the bistable device is reflective, and the signal is routed to the output; when the control beam is on [Fig. 5(b)], the device is in the low-reflection state, and the signal beam is mostly absorbed.

We can transform this operation mode into active switching by setting the high-level control power closely below the switching threshold, as shown in Fig. 5(c). If the additional signal power exceeds the threshold, the device is switched by each data-bit change. So the modulation of the signal is transferred onto the reflected control beam, which is considered as the output in this case. Because the

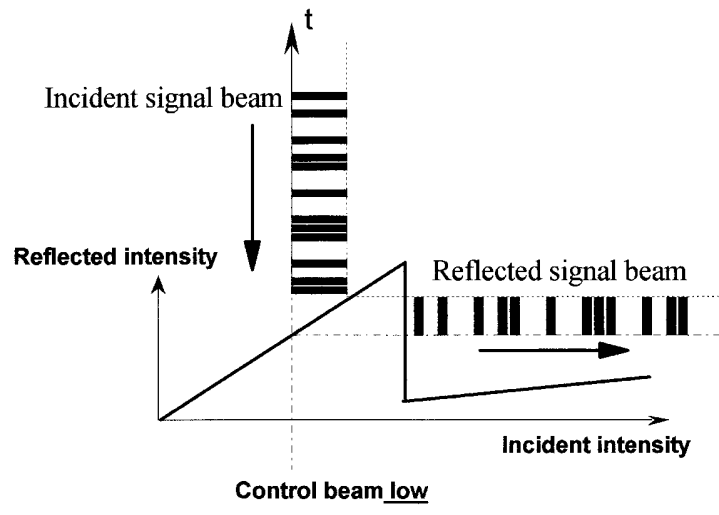

(a)

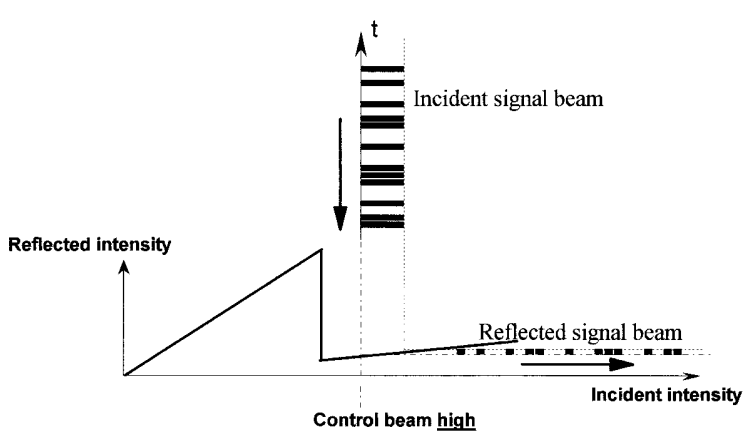

(b)

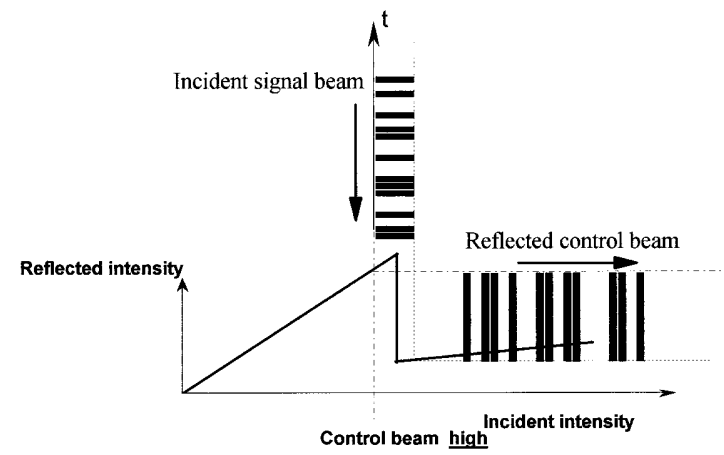

(c)

Fig. 5. Circuit switching of data that are controlled by the holding-beam power: (a) transmitting state when control power is below the threshold, (b) absorbing state when control power is above the threshold, and (c) active switching with the signal modulation transmitted to the control beam if the control power is just below the threshold; output is low if the input signal is high, and output is high if the input signal is low.

control beam is more intense than the signal beam, data amplification and logic-level restoration can be obtained. Setting the control beam to a lower or a higher value disables the operation.

\section{Experimental Studies}

\section{A. Setup}

The layout of the experiment is shown in Fig. 6 (the figure and the caption apply to packet switching; some minor changes for circuit switching experiments are explained in the text). The experimental arrange- 


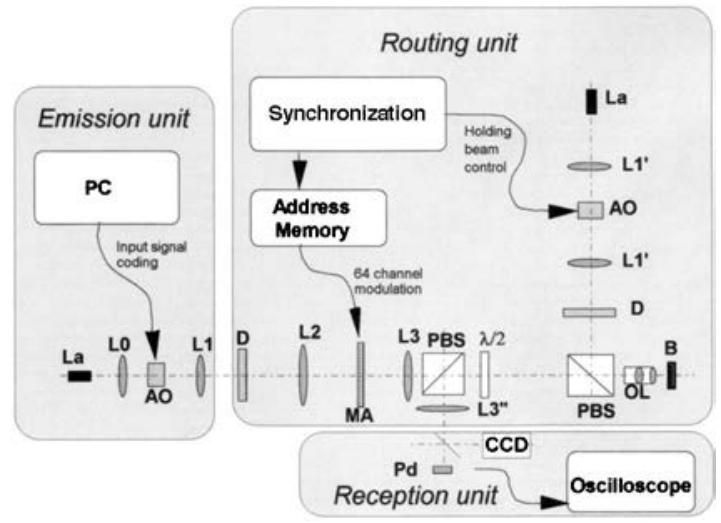

Fig. 6. Optical setup of the demonstrator system: La, laser; AO, acousto-optical modulator; OL, objective lens; L, Lens; D, Dammann grating; MA, MQW electro-optical modulator array; Pd, photodiode; $\lambda / 2$, half-wave plate; PBS, polarizing beam splitter; $\mathrm{B}$, MQW optical bistable device.

ment involves three basic parts: the emission, the routing, and the reception units. The task of the emission unit is to generate the input signal by amplitude modulation of the continuous beam from an Xcavity Ti:sapphire laser by the use of a computercontrolled acousto-optical modulator. Two types of input signals can be delivered by this unit: a continuous bit stream for circuit-switching experiments and a form described in Subsection 3.A that is suitable for data packets. The issued data can be both recurrent for the tuning and testing of the setup and random for the demonstration of the realistic routing operation.

As it enters the routing unit, the input beam is divided into $8 \times 8$ subbeams by diffraction on a binary fan-out grating (Dammann grating) ${ }^{16,17}$ to illuminate the 64 pixels of the electroabsorption modulator array. The modulator array is driven by a small electronic circuit that contains eight pieces of 8-bit-wide and 16-bit-deep electrically programmable read-only memories to store the 64 channel addresses. Each of the 64 memory outputs is connected to the individual control electrode of the corresponding modulator pixel through a buffer that delivers the $6-\mathrm{V}$ swing required by the modulator. The common electrode is set to $12 \mathrm{~V}$ so that the $\mathrm{p}-\mathrm{i}-\mathrm{n}$ structure modulator pixels are backward biased to 6 $\mathrm{V}$ and $12 \mathrm{~V}$ in the transmitting and the absorbing states, respectively. The operation of the circuit is triggered by the synchronization pulse that precedes each packet in order to obtain perfect synchronization between the packet address and the modulation. The modulator array is imaged upon the bistable cavity by a telescopic pair of lenses (L3 and OL of Fig. 6) with a demagnification of 5 . Because we used planar (nonpixellated) bistable devices in this experiment, the spot array from the modulator array must be aligned only with respect to the array of holdingbeam spots.

The holding beam-issued from the same laser as the signal beam for the present experiment-is con- trolled by another acousto-optical modulator. This modulator serves for the temporary reduction of the holding power before the arrival of the packet to reset the bistable elements into the high-reflectivity state. Its operation is synchronized by the same electronic circuit described above. The holding beam is then transformed into an array of 64 beams by means of a fan-out grating similar to the previous one. A polarizing beam splitter directs the beams toward the bistable cavity where, as was said above, they are superimposed upon the signal beam spot array. The holding beams reflected by the cavity propagate back following the same path without disturbing the outputs.

A part of the reflected signal power is conducted to the reception unit by means of a half-wave plate and a polarizing beam splitter. By the rotation of the wave plate the power coupled out can be varied from $0 \%$ to $25 \%$ of the incident signal. The separation of the output beam without any losses can be done only by use of a Faraday rotator and a half-wave plate placed between the two polarizing beam splitters.

A single photodiode was used as a detector on individual channels in the high-data-rate experiments. A CCD camera was mounted parallel to the outputs to facilitate the tuning and the alignment of the system.

\section{B. Packet-Switching Experiments}

Single-channel operation of the demonstrator system has been presented elsewhere. ${ }^{18}$ Therefore we give only a brief summary to show differences and similarities with the attempted parallel operation.

The combination of address decoding and latching was first demonstrated in a single channel of the system with the following parameters: packet length of 80 bits, bit rate of 20 Mbits/s, bistability contrast of $\sim 1.5$. All 60 defect-free channels of the system could be operated this way. Figure 7 shows the operation of channel 63 with two different packets, one addressed to this output and the other addressed to output 61 . The peak power incident upon the single bistable element was $11 \mathrm{~mW}(10 \mathrm{~mW}$ of holding and $1 \mathrm{~mW}$ of signal power). The maximum usable packet length was limited by parasitic commutations that occur during latching owing to power fluctuations of the laser beam. We were able to maintain error-free operation for several minutes without tuning, but the system then became unstable, probably because of thermal drifts on the active components. Further work was carried out toward a threefold objective: enhance the switching contrast, improve the system stability, and decrease the required optical power.

A switching contrast of 2 and a minimum operating power of $3.5 \mathrm{~mW}$ per bistable element was obtained with a thorough optimization of the working conditions of the bistable cavity (the wavelength, the working point, the temperature, and the incident spot size). To increase the stability and the maximum packet length that can be transmitted without parasitic commutation, we reduced the power fluctuations by better tuning the laser and improved the thermal 


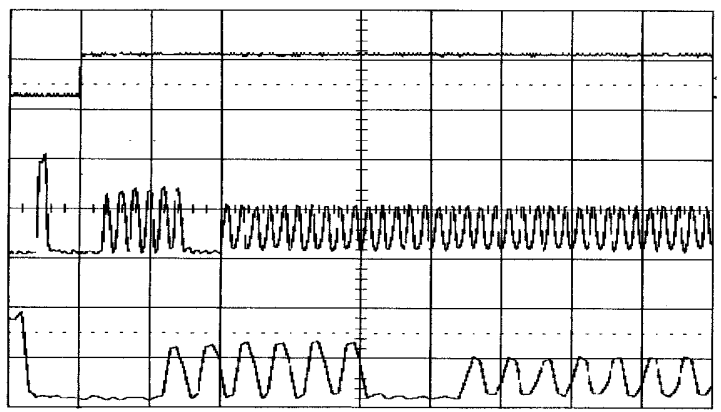

(a)

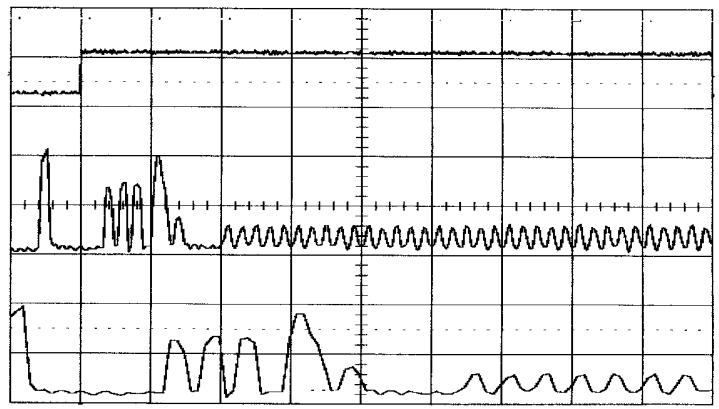

(b)

Fig. 7. Demonstration of single-channel operation of the system. Channel 63 is used with two different packets: (a) one addressed to this output and (b) the other addressed to output 61. The first oscilloscope trace corresponds to the holding-beam power and the second and the third show the output of the system, the latter with a zoom on the header of the packets. The packet length is 80 bits, the bit rate is $20 \mathrm{Mbits} / \mathrm{s}$, and the bistability contrast is $\sim 1.5$.

control of the bistable device using a custom temperature regulation circuit (the modulator array could not be temperature regulated because it operates in transmission). Thus an acceptable noise level ( $0.7 \%$ rms noise power) was obtained at the output of the emission unit. This could have been reduced further only by means of active power regulation of the laser source, at the price of considerable power losses.

With these parameters the system operated at packet lengths of 400 bits. With the same settings but at $10-\mathrm{mW}$ incident power the packet length attained 1600 bits without any observable error in address decoding and latching. The system operated even at 8-kbit data string length with an error rate of a few percentage points.

A trade-off was observed between the noise robustness and the minimum power consumption criteria: Reducing the operating power increased the instability of the system. This trade-off is essentially due to the narrowing of the relative hysteresis width of the bistable devices that occurs concurrently with the threshold reduction when one decreases the detuning from the Fabry-Perot resonance. However, threshold powers below $4 \mathrm{~mW}$ had to be retained in view of the planned parallel operation of the 64 channels because the total laser power available was limited to $1 \mathrm{~W}$.

To demonstrate simultaneous operation of several channels of the system, one must select a working

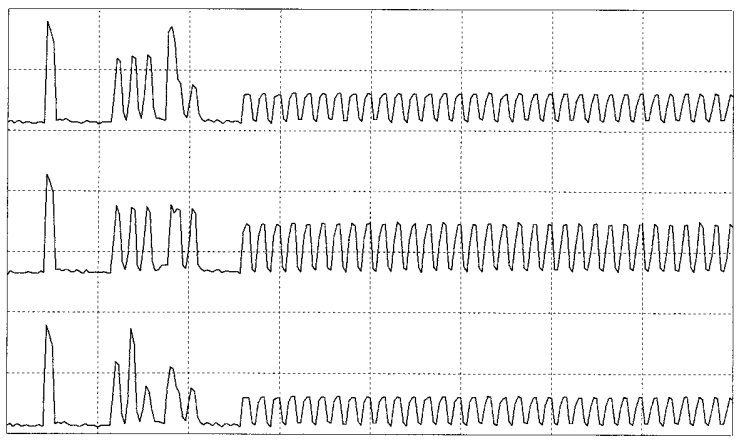

Fig. 8. Output signals obtained by the simultaneous self-routing operation of three channels of the setup. We recorded the three traces one after the other by moving the single-channel receiver into the different output beams. The packet is addressed to output 59 and the three observed outputs are channels 63,59 , and 47.

point on the cavity at which the threshold values of several bistable elements are coincident. A two-step procedure was implemented to achieve this purpose; it made use of a uniform array of 64 light spots with continuously variable power. First, the blinking of all 64 spots on the cavity is observed by the CCD camera while the incident power is changed gradually. Because the reflected power changes suddenly during the switch on and the switch OFF, the number of spots that switch simultaneously can be evaluated. This number is then maximized in function of the lateral position, the temperature of the sample, and the wavelength. The second step is to equalize accurately the real switching thresholds of these elements by fine-tuning the same parameters while detecting their superposed responses during highfrequency operation. By this method a maximum of five channels was found that presented the same switching thresholds within $\pm 20 \%$.

Keeping the parameter settings obtained by the above procedure, we apply the real input signals, i.e., the signal and the holding beams, with the modulation defined in Subsection 3.A. With suitable tuning of the holding and signal powers, simultaneous operation of up to three channels was observed (see Fig. 8).

The stability in this experiment was quite poor; correct operation could not be obtained for data string lengths that exceeded 400 bits. The low level of stability and parallelism is due to the nonuniformity of active and passive devices and the remaining power fluctuations of the beams. According to the tolerance analysis in Ref. 19, error-free parallel operation of all the channels of the demonstrator would require the following goal parameters (for comparison, the same parameters of the present system are given in parentheses): spatial fluctuations less than $\pm 4 \%$ $( \pm 20 \%)$ for the bistable devices, $\pm 5 \%( \pm 17 \%)$ for the modulator array, and $\pm 3 \%$ ( $\pm 8 \%$ ) for the remaining passive optical system (mainly caused by the beamarray generator); relative hysteresis width of $50 \%$ $(15 \%)$; a contrast ratio of $\sim 10(2)$; and a rms noise power below $0.5 \%(0.7 \%)$. (Note that the contrast ratio has to be increased further if a fan-in operation 


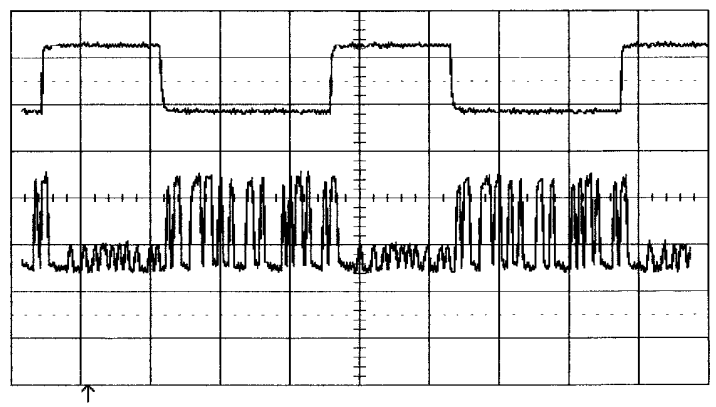

Fig. 9. Control signal (upper trace) and output (lower trace) observed in the circuit-switching experiment.

is to be implemented between the outputs, as for the crossbar switch described in Subsection 5.A.)

The most demanding of these goal parameters is the high homogeneity required for the active devices. To circumvent this problem, we can achieve nonuniformity compensation by tuning the power level incident to each bistable pixel near the optimal value required by that particular pixel. This can be implemented in small systems by use of microlaser arrays with individual power control of each element or a single laser source with a weighed array illuminator. Of course, this solution is impractical for highly parallel systems where the problem can be only slightly alleviated by the use of sources [e.g., verticalcavity surface-emitting laser (VCSEL) arrays] that present similar long-scale wavelength or power dispersion as bistable microresonators.

The clock frequency of the system is fixed at 20 $\mathrm{MHz}$ owing to our experimental means and is not related to device response times, which are of the order of nanoseconds. The operation of the system at address bit periods considerably shorter than 50 ns, however, would require higher signal powers because of the critical slowing down ${ }^{20}$ of the bistable devices at low switching powers. The data bit rate can be increased independently of device limitations because the system is entirely transparent to data.

\section{Circuit-Switching Experiments}

To implement this operating mode, the setup of Fig. 6 was rearranged: The holding and signal beams were interchanged to control each holding beam individually by the modulator matrix. Figure 9 shows the operation of this scheme: When the control beam (upper trace) is OFF, the signal (lower trace) is reflected; when the control beam is ON, the signal beam is mostly absorbed. In this operating mode the contrast ratio can be improved up to a value of 4 while the threshold power can be as low as $1 \mathrm{~mW}$, in contrast with $3.5 \mathrm{~mW}$ in the bistable mode.

This scheme presents operating conditions that are much less severe than the latching operation if the signal beam is weak compared with the control power (the signal-to-control power ratio is typically $1 / 5$ in the experiments). This results in increased robustness against noises and nonuniformities. Our experimen-

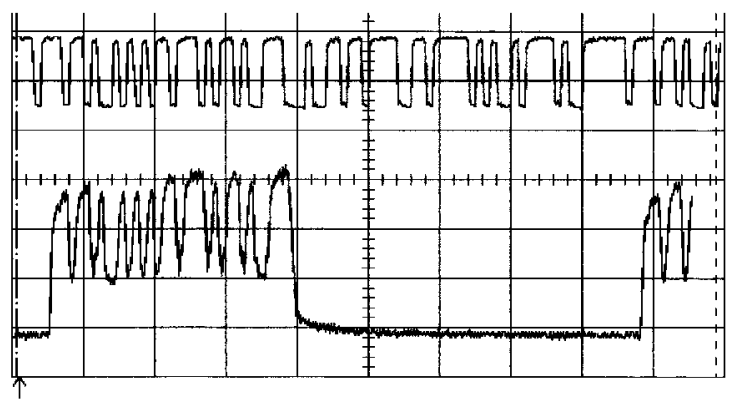

Fig. 10. Input (upper trace, with inverted polarity) and output (lower trace) observed in the active switching experiment, with control-beam modulation similar to that of Fig. 9.

tal results confirm this expectation: 37 channels out of 64 could be operated simultaneously with a contrast ratio superior to 2 . The remaining 27 outputs presented some modulation as well, but with a lower contrast ratio. Neither noise-induced commutations nor long-term instabilities were observed.

For the demonstration of active switching with signal reshaping and amplification according to the scheme of Fig. 4(c), the setup remains unchanged except that the reflected control beams are used as outputs. Figure 10 shows the input and the output signals obtained by single-channel operation of the system. The input data is transferred to the output with an amplification greater than 1 when the control beam is on, and there is no output when it is off.

This operation mode requires data-rate switching of the bistable element, which means that the throughput of the system is limited by the rise and fall times of the bistable device. These were of the order of $20 \mathrm{~ns}$ in this experiment, with an input power of $2 \mathrm{~mW}$ in the control beam and $1 \mathrm{~mW}$ in the signal beam. This is evidence of the critical slowing that occurs when the bistable device works at low excess powers beyond the threshold value. This effect manifests itself as a kind of limited gain-bandwidth product in this experiment.

The gain is limited by two other factors as well: First, the slope and the contrast ratio of the steplike response curve give an upper limit for the gain of a single device that operates in noiseless environment. Second, the noise margins for temporal and spatial fluctuations become tighter and tighter when they increase the gain. This indicates a clear trade-off between the gain and the tolerated overall signal-tonoise ratio (for a thorough tolerance analysis see Ref. 19). These limitations do not allow the system to be used as a high-bandwidth amplifier, but this gainbandwidth product can be sufficient for implementing moderate data-rate cascadable logic gates or switching elements.

\section{System Evolution Possibilities}

\section{A. $N$ to $M$ Switching Network}

Both the self-routing and the direct control schemes are readily extensible to $N \times M$ crossbarlike switch- 
ing networks. One possible architecture is based on optical vector-matrix multiplication: A row vector of $N$ input beams multiplied by the $N * M$ control matrix results in a column vector of $M$ output beams. ${ }^{21,22}$ A nonzero element (i.e., a transmitting pixel) in row $i$ and column $j$ of the matrix establishes a connection between input $i$ and output $j$. To implement selfrouting, we can replace the control matrix with the ensemble of an $N \times M$ modulator array and an $N \times$ $M$ bistable device array. Each of the $N$ input beams carries a different signal, organized in the packet format described above, with a ( $\left.\log _{2} M\right)$-bit-long Manchester-coded destination address. Each beam is split into $M$ parts by a one-dimensional fan-out element so that it illuminates the corresponding column of modulator elements. In each column of the system an optical address recognition takes place on the same principle as in the 1-to-64 switch. As a result, one bistable element in each column stays in the high-reflectivity state, providing the nonzero elements of the control matrix. The reflected beams from different columns but identical rows of the bistable array are then collected by a fan-in element to form the $M$-element output array. In such a system, the arrival of the signal packets can be completely asynchronous because the address-decoding operations are self-synchronized and independent from one column to another.

The scalability of this scheme is limited by the power losses encountered during the fan-out and fan-in operations. ${ }^{23}$ It is obvious that fan-out by a factor of $M$ introduces an $M$-fold power loss of the signal beam. It is less obvious that fan-in suffers from the same trouble in most cases. From the conservation of étendue (also referred to as the constant radiance theorem) it follows that the optical fan-in of $N$ mutually incoherent beams introduces an $N$ fold power loss if the resulting single beam has a cross-sectional area and a numerical aperture the same as the input beams. We can avoid this loss by increasing the étendue of the output port by a factor of $N$ by using larger-numerical-aperture optics, largerdiameter detectors, or suitable multimode fibers on the output side. In this case, however, the cascadability of several identical systems will be prohibited.

Another restriction on the number of signal beams that can be incoherently added comes from noise accumulation: For the optical crossbar, the theoretically blocked signal that, in fact, passes with nonzero power must be considered as noise. Consequently, the signal-to-noise ratio after the $N$-fold fan-in is smaller than the power-of-1 passed signal divided by the sum of the powers of the theoretically blocked signals, that is, the extinction ratio (switching contrast) of the elementary switches divided by $N$. Therefore, to realize an $N \times M$ crossbar, we need bistable devices with contrast ratios significantly larger than $N$.

\section{B. Miniaturization of the System}

To conceive a practical usable system or even a valuable demonstrator, we must consider system integra-

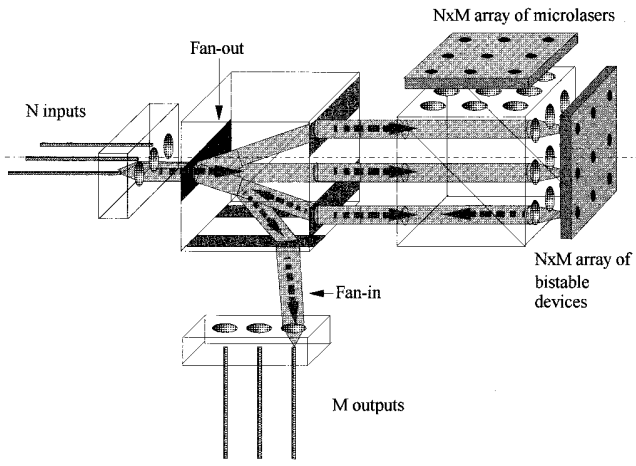

Fig. 11. Miniaturized version of the $N$ to $M$ crossbar switch by use of the optical matrix-vector multiplication scheme.

tion and miniaturization in order to reduce the dimensions, the cost, and the power consumption. Figure 11 shows an outline for the $N$-to- $M$ version of the switch. One main difference with the present setup would be the substitution of the T:sapphire laser and the modulator array by a single device-a VCSEL array.

The use of VCSEL's is promising from the miniaturization point of view, but their compatibility with recent optical bistable device arrays requires further study concerning stability and homogeneity of wavelength, power, and polarization. Note that by use of VCSEL arrays with individual driving electronics for each microlaser an active nonuniformity compensation can be achieved. This means that the power level incident on each bistable device can be tuned individually to near the optimal value required by that particular device. Each VCSEL of the $N \times M$ array generates a holding beam for the corresponding bistable element. When we implement the selfrouting operation with this version of the system, the reference addresses for address comparison are carried by the holding beams as a small signal added to the constant holding power.

Figure 12 shows the operation of the address de-

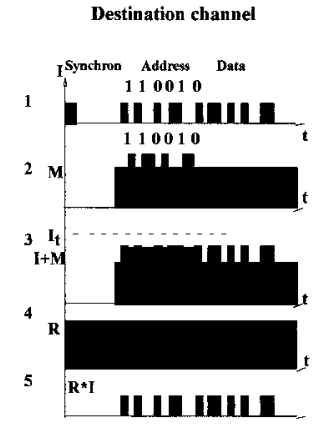

(a)

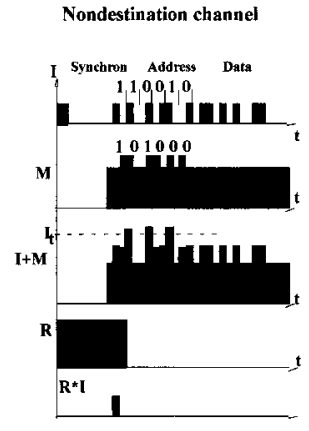

(b)
Fig. 12. Signal diagram of the self-routing operation with the addition of the destination address to the reference address issued by a VCSEL: 1 , input signal; 2 , holding beam with the superposed reference address; 3 , incoherent sum of the input and holding beams; 4, reflectivity of the corresponding bistable element; 5, signal reflected by the bistable element (equals the output). 
coding in this case. The main difference from the original scheme is that here we do not accomplish address identification by multiplying the destination address by the reference address but by incoherently adding the Manchester-coded destination address (carried by the input signal) to the inversely coded reference address (carried by the holding beam). Thus any mismatch between the two addresses produces a double-power switching pulse for the bistable element. With this method there is no need for emitting the data at a power level lower than the header address. As there is no difference between address and data pulses, the purely binary input signal is much easier to generate, and the first data bits of a packet can be used directly as address bits for upcoming switching stages in case of cascading several switches.

The inputs and the outputs of the setup are implemented by micro-optical fiber connectors with an integrated collimating microlens for each fiber. This component is under development in our laboratory. ${ }^{24}$ The setup contains additional passive optical elements such as two polarizing beam splitters, two arrays of deflecting microprisms or holograms, a onedimensional fan-out grating, and a half-wave retardation plate. The overall volume can be a few cubic centimeters for an 8-input-8-output switch, not including the driving electronics for the microlaser array.

\section{Conclusion}

We have demonstrated experimental results of the operation of a 1-to-64 free-space photonic switch in both packet-switching and circuit-switching environments. We implemented packet switching by optical decoding of the packet header, using the latching operation of bistable devices. Although almost all channels of the setup could be operated individually, simultaneous operation of only three of them was demonstrated. This is mainly due to the high spatial fluctuations of certain device parameters such as the bistable thresholds and the modulator transmissions.

The system stability was limited by the temperature drift of the modulator element and the noise from the laser source. To enhance the available parallelism and the system stability, further experiments will make use of new generation active devices that present better uniformities and possibly lower temperature drifts. Parameters that determine the overall system tolerances (for example, the hysteresis width or the modulation contrast) can also be improved greatly. Compensation of the overall nonuniformities is envisioned by means of individual tuning of the holding powers.

In the circuit-switching mode, direct optical control of the switching nodes was demonstrated. As this scheme is much more tolerant of spatial and temporal fluctuations, parallel operation of 37 channels could be achieved. Signal amplification has also been demonstrated within this mode. Further experiments will concentrate on the production of a miniaturized implementation of the described system to obtain a usable building block or interconnection networks in supercomputers or telecommunication machines.

This study was supported by the French Ministry of Research under the project Matrices Optoélectroniques pour le Traitement du Signal (MOTS). We acknowledge the contributions of R. Azoulay, O. Hellesoy, B. Jouault, and D. Marie-Noël to this work.

\section{References}

1. F. B. McCormick, T. J. Cloonan, A. L. Lentine, J. M. Sasian, R. L. Morrison, M. G. Backman, S. L. Walker, M. J. Wojcik, S. J. Hinterlong, R. J. Crisci, R. A. Novotny, and H. S. Hinton, "Five-stage free-space optical switching network with FETSEED smart pixel arrays," Appl. Opt. 33, 1601-1618 (1994).

2. P. R. Prucnal and P. A. Perrier, "Self-routing photonic switching with optically processed control," Opt. Eng. 29, 170-182 (1990).

3. M. Fraces, "Multiprocessor based on an optical crossbar network," in Optical Computing '88, P. Chavel, J. W. Goodman, and G. Roblin, eds., Proc. SPIE 963, 36-40 (1988).

4. H. J. White, N. A. Brownjohn, C. Stace, G. M. Proudley, A. C. Walker, M. R. Taghizadeh, B. Robertson, C. P. Barrett, and M. J. Birch, "Practical demonstration of a freespace optical crossbar switch," in Optical Design for Photonics, Vol. 9 of 1993 OSA Technical Digest Series (Optical Society of America, Washington, D.C., 1993), pp. 140-143.

5. S. D. Smith, "Lasers, nonlinear optics and optical computers," Nature 316, 319-324 (1985).

6. D. Pellat, R. Azoulay, J. L. Oudar, "Relationship between hysteresis width, nonlinear index saturation and cavity finesse in GaAs/AlGaAs multiple quantum well bistable microcavities" in Technical Digest of the 5th European Quantum Electronics Conference, IEEE Conf. Proc. 94, 323-326 (1994).

7. H. Ambrosius and C. J. Van der Poel, "Strained layer quantum well laser diodes grown in a Planet MOVPE reactor," in Proceedings of the 1993 MO-VPE Workshop (1993).

8. D. Frank and B. Wherrett, "Optical bistability in cylindrical pixels," Opt. Eng. 26, 53-61 (1987).

9. T. Rivera, F. R. Ladan, A. Izrael, R. Azoulay, R. Kuszelewicz, and J. L. Oudar, "Reduced threshold all-optical bistablity in etched quantum well microresonators," Appl. Phys. Lett. 64, 869-871 (1994).

10. C. Bagnoud and N. Collings, "Study of a bistable GaAs FabryPerot device for operation in array format," Opt. Commun. 114, 151-154 (1995).

11. B. G. Sfez, J. L. Oudar, J. C. Michel, R. Kuszelewicz, and R. Azoulay, "High contrast multiple quantum well optical bistable device with integrated Bragg reflectors," in Proceedings of the Conference on Nonlinear Dynamics in Optical Systems (Afton, 1990).

12. J. P. Schnell, J. Raffy, J. P. Pocholle, A. Delboulbe, G. Dutrey, J. Lehoux, M. Werner, M. Papuchon, and J. P. Huignard, "Two-dimensionnal multiquantum well spatial light modulators for interconnections and optical processing," in Spatial Light Modulators and Applications, Vol. 14 of 1990 OSA Technical Digest Series (Optical Society of America, Washington, D.C., 1990), pp. 60-64.

13. D. A. B. Miller, D. S. Chemla, T. C. Damen, A. C. Gossard, W. Wiegmann, T. H. Wood, and C. A. Burrus, "Band-edge electroabsorption in quantum well structures: the quantum confined Stark effect,” Phys. Rev. Lett. 53, 2173-2175 (1984).

14. C. C. Barron, C. J. Mahon, B. J. Thibeault, and L. A. Coldren, "Design, fabrication and characterisation of high speed asymmetric Fabry-Perot modulators for optical interconnect applications," Opt. Quantum Electron. 25, 885-898 (1993).

15. G. S. Buller, C. R. Paton, S. D. Smith, and A. C. Walker, 
"All-optical routing networks based on bistable interferometers," Appl. Phys. Lett. 53, 2465-2467 (1988).

16. H. Dammann and K. Görtler, "High-efficiency in-line multiple imaging by means of multiple phase holograms," Opt. Commun. 3, 312-315 (1971).

17. J. L. Tribillon, "Paraxial calculation of Dammann-type pupils, allowing the multiplication of images: exact solution and error calculation," Pure Appl. Opt. 3, 389-412 (1994).

18. P. Koppa, P. Chavel, J. L. Oudar, R. Kuszelewicz, J. P. Schnell, and J. P. Pocholle, "Optical self routing using address decoding,” J. Phys. III France 4, 2405-2411 (1994).

19. P. Koppa, P. Chavel, J. L. Oudar, R. Kuszelewicz, and E. Lugagne-Delpon, "Uniformity and noise limitations of free space optical computing and switching systems based on arrays of optical bistable devices," Pure Appl. Opt. 5, 899-917 (1996).

20. J. L. Oudar, B. G. Sfez, R. Kuszelewicz, J. C. Michel, and R.
Azoulay, "Optical nonlinearities of GaAs-based epitaxial structures for all-optical switching," Phys. Status Solidi B 159, 181-189 (1990).

21. J. W. Goodman, A. R. Dias, and L. M. Woody, "Fully parallel, high speed incoherent optical method for performing discrete Fourier transforms," Opt. Lett. 2, 1-3 (1978).

22. A. A. Sawchuk, B. K. Jenkins, C. S. Raghavendra, and A. Varma, "Optical matrix-vector implementation of crossbar interconnection networks," in Proceedings of the 1986 International Conference on Parallel Processing, (IEEE, New York, 1986).

23. J. W. Goodman, "Fan-in and fan-out with optical interconnections," Opt. Acta, 32, 1489-1496 (1985).

24. M. Kufner, S. Kufner, P. Chavel, and M. Frank, "Monolithic integration of microlens arrays and fiber holder arrays in PMMA with fiber self-centering,” Opt. Lett. 20, 276-278 (1995). 